\title{
Optimal Adaptive Inertial Droop Control-Based Power System Frequency Regulation via Wind Farms
}

\author{
Yijun Chen ${ }^{1}$, Bo Yang ${ }^{1}$, Yiming Han ${ }^{1 *}$, Tingyi $\mathrm{He}^{2}$, Peng $\mathrm{He}^{2}$, Xian $\mathrm{Meng}^{2}$ and Xin $\mathrm{He}^{2}$ \\ ${ }^{1}$ Faculty of Electric Power Engineering, Kunming University of Science and Technology, Kunming, China, ${ }^{2}$ Electric Power \\ Research Institute of Yunnan Power Grid Co., Ltd., Kunming, China
}

Wind farm-based frequency regulation of the power system has progressively attracted more attention owing to its higher power generation capacity. Among which the step start-up and adaptive inertial droop control of wind turbines with a maximum power point efficiently regulate the system frequency via fully utilizing the rotational kinetic energy. Besides, the coefficients of adaptive droop control and virtual inertial control will facilitate a significant influence on the frequency support performance and operation status of wind turbines for the power grid. To obtain optimal control parameters, a parameter optimization framework of a step start-up adaptive inertial and droop controller combined with the CGO algorithm is proposed, whose

OPEN ACCESS

Edited by:

Bin Zhou,

Hunan University, China

Reviewed by:

Xuehan Zhang,

Korea University, South Korea

Xiaoshun Zhang,

Shantou University, China

${ }^{*}$ Correspondence:

Yiming Han

373482753@qq.com

Specialty section:

This article was submitted to

Process and Energy Systems

Engineering,

a section of the journal

Frontiers in Energy Research

Received: 21 November 2021

Accepted: 03 December 2021

Published: 12 January 2022

Citation:

Chen Y, Yang B, Han Y, He T, He P,

Meng $X$ and $\mathrm{He} X$ (2022) Optimal Adaptive Inertial Droop Control-Based Power System Frequency Regulation

via Wind Farms.

Front. Energy Res. 9:819398. doi: 10.3389/fenrg.2021.819398 effectiveness is verified by a three-area four-terminal VSC-MT-HVDC-based WFs and AC system in MATLAB/Simulink. As a result, a set of parameters with a satisfactory and comprehensive dynamic control performance can be acquired by the proposed method under both $200 \mathrm{MW}$ and $300 \mathrm{WM}$ load increases compared with the trial-and-error approach.

Keywords: wind farms, frequency regulation, droop control, virtual inertial control, chaos game optimization

\section{INTRODUCTION}

In recent years, for the purpose of reducing dependence on fossil fuels, the electric power structure needs to shift more toward renewables to facilitate energy transformation (Zhang et al., 2015; Hou et al., 2017; Meng et al., 2021). Consequently, renewables (Chen et al., 2018; Li et al., 2020; Pabitra and Abhik, 2020; Zhao et al., 2021a) (e.g., solar, wind, hydro energy, etc.) have been wildly developed around the world, upon which the wind energy is increasingly emerging as one of the most mature, promising, and representative renewables, thanks to its unique superior, i.e., clean, reproducible, stable, well-stocked (Liu et al., 2021), and widespread (Díaz and Guedes Soares, 2020).

Yunnan, located in the southwest region of China, is equipped with abundant hydropower and wind power resources. However, the high proportion of installed hydropower also poses new issues for the Yunnan power grid. The water hammer effect (WHE) (Vereide et al., 2017; Al BkoorAlrawashdeh et al., 2021) is a momentous characteristic of the hydraulic turbine governing system, which produces negative damping to the system and thus brings about system oscillation. Furthermore, a typical WHE curve is illustrated in Figure 1 when the degree of guide vane opening of large hydropower units is increased to handle the augment in load demand in the Jin'anqiao hydropower station of Yunnan. It can be

Abbreviations: AC alternating current; CGO chaos game optimization; DFIG-WT doubly fed induction generator-based wind turbine; DC direct current; FFD first frequency drop; MPPT maximum power point tracking; MPP maximum power point; SFD secondary frequency drop; TFD tertiary frequency drop; VSC-MT-HVDCs multi-terminal high voltage direct current system; WHE water hammer effect; WFs wind farms; without FSC without WFs participating in frequency regulation. 


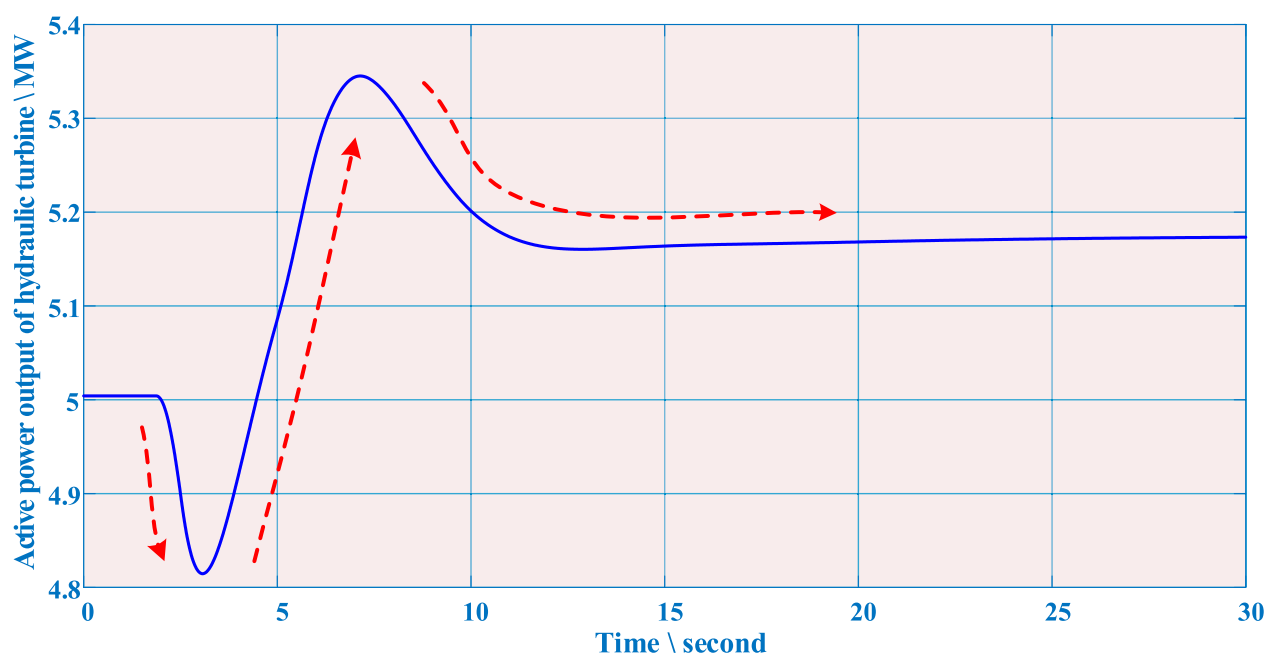

FIGURE 1 | Water hammer effect under large hydropower units removal in the Jin'anqiao hydropower station of Yunnan.

transparently seen that WHE causes turbines to produce antithetical performance relative to actual control from Figure 1. That is to say, the output power of turbines will temporarily drop before increasing, which gravely threatens the security and stability of power grid operation, particularly when a large load disturbance occurs in a power system with a high hydraulic turbine capacity. It is noted that the participation of wind farms (WFs) in frequency regulation is an alternative and effective solution to address this tricky obstacle and achieve active power stabilization.

However, WFs are mostly distributed far from load centers and need to utilize the voltage source converter-based multiterminal high voltage direct current system (VSC-MT-HVDCs) to implement electricity transmission (Renedo et al., 2016; Gu et al., 2021). On account of decoupled operation between rotor speed and power system frequency, the doubly fed induction generator-based wind turbine (DFIG-WT) does not directly provide dynamic frequency support for the power grid similar to the inertial response characteristic of synchronous generators (Wang-Hansen et al., 2013; Xiong et al., 2021). Power system inertia is decreased owing to the access of DFIG-WT with a highpenetration level in the power grid, which also raises the frequency regulation pressure (Wang et al., 2019). With a view that the power system frequency fluctuation perhaps causes some serious consequences (Wei Yao et al., 2015; Yang et al., 2015; Golpira et al., 2016; Xiong et al., 2020), e.g., affecting industrial production, triggering relay protection equipment, and even causing frequency collapse phenomena, it is enormously imperative to energetically explore corresponding strategies to realize frequency support from WFs for a secure and stable operation of the alternating current (AC) system.

In the past few decades, multifarious frequency regulation control strategies of the AC system through WFs combined with VSC-MT-HVDCS have been developed (Huang et al., 2021; Zhou et al., 2021), which can be divided into two major categories in general, i.e., indirect and direct methods (MacDowell et al., 2015; Liu et al., 2020; Zhao et al., 2021b). Specifically, indirect active power control introduces large-capacity capacitors in the flexible direct current (DC) transmission system to achieve frequency regulation of the AC side by controlling the voltage of the DC system to release capacitance energy (Zhu et al., 2013; Wen et al., 2016; Gan et al., 2019; Jami et al., 2020; Kadri et al., 2020; Yang et al., 2022). Nevertheless, lack of economy is a pivotal ingredient impeding the widespread application of the indirect method. Comparatively, another one is accomplished by deloading, the discharging kinetic energy of the wind turbine rotor, adjusting pitch angle, etc., (Navalkar et al., 2015; Fu et al., 2017; Boyle et al., 2021; Xiong et al., 2021), whereas deloading operation strategy reserves backup power (Yao et al., 2019) but seriously remedies the efficient engagement of wind resources. Besides, frequently controlling the pitch angle can increase mechanical stress on the wind turbine blades and subsequently endanger the safety of wind turbines (Varzaneh et al., 2014). Accordingly, to implement friendly grid-connected WFs with a fast response to system frequency variation, it is a popular and promising approach to adequately employ the rotational kinetic energy and backup power of wind turbines to simulate the inertial response and primary frequency regulation ability of synchronous turbines. A fuzzy PID control based on maximum power point tracking (MPPT) control is suggested in the literature (Varzaneh et al., 2014) in such a manner that the kinetic energy stored in the rotor inertial is used to control the output power of wind turbines. However, a fuzzy PID control requires real-time-modulating PID parameters according to current system states, and thus, its applicability encountering different scenarios needs to be further validated. The literature reports (Surinkaew and Ngamroo, 2014) considered the uncertainties of wind power and load, which proposed a novel coordinated robust control method for DFIGs and synchronous generators to stabilize system oscillation and adopt an improved firefly algorithm to optimize parameters of the controller. Moreover, a deloading control strategy based on the available margin is developed in reference (Vidyanandan and Senroy, 2013) to furnish primary 
frequency support for the power system. It is noted that the transitory frequency support perhaps induces the secondary frequency drop (SFD) phenomenon during the recovery process of the wind turbine speed, which is probably worse than the first frequency drop (FFD) if the active power is seriously insufficient. As a consequence, reference (Xiong et al., 2021) attempted to realize appropriate frequency support and alleviate SFD via a two-level combined control strategy of VSC-MT-HVDC-integrated offshore WFs. Nevertheless, the trial and error method was adopted to determine the corresponding parameters of this control mechanism, which extremely imposed a burdensome mission on power grid operators and did not guarantee the control accuracy and stability. Whereupon, for tackling the aforementioned shortcomings, a parameter design framework of a step start-up adaptive inertial droop controller via chaos game optimization (CGO) (Talatahari and Azizi, 2020) is developed to automatically grope the satisfactory control performance and then decidedly mitigate superfluous labor in the new operating circumstances similar to the WHE phenomenon of the Yunnan grid in this article.

Synoptically, this article incorporates five sections as follows. First, Section 1 chiefly introduces the research background, conducts a literature review, and indicates the intentions of this work. Section 2 concisely describes the step start-up adaptive inertial and droop control scheme. Then, the parameter design framework proposed in this article is exhibited in Section 3in detail. The verifications of the presented method are executed in Section 4. Finally, Section 5 thoughtfully illustrates several conclusions and perspectives.

\section{STEP START-UP ADAPTIVE INERTIAL AND DROOP CONTROL SCHEME}

The step start-up adaptive inertial and droop control scheme is demonstrated in Figure 2 for a three-area four-terminal VSCMT-HVDC-based WFs and AC system (Vennelaganti and Chaudhuri, 2018; Xiong et al., 2021). It is noted that the wind turbines are categorized into two clusters according to their rotor speeds to orderly launch after the frequency events (Xiong et al., 2021). Besides, the rotor speed of each wind turbine in both WF 1 and WF2 is determined as $0.90 \mathrm{pu}, 0.95 \mathrm{pu}, 0.85 \mathrm{pu}, 1.00 \mathrm{pu}$, and $1.05 \mathrm{pu}$ in sequence, and the discrimination threshold of the two clusters is settled as $0.9 \mathrm{pu}$. Emphatically, the step start-up adaptive inertial and droop control scheme is only applied in WF 1, where all wind turbines operate in MPPT situations. Therefore, the output power reference value $P_{\text {ref, } i}$ of the $i$ th wind turbine in WF1 includes three items, i.e., maximum power point (MPP) $P_{m p p, i}$ under the rotor speed $\omega_{i}$, droop control, and virtual inertial control, which can be calculated as follows:

$$
\begin{gathered}
P_{\mathrm{ref}, i}=P_{\mathrm{mpp}, i}+k_{\mathrm{dr}, i} \cdot\left(f_{\mathrm{AC}}-f_{\mathrm{ref}}\right)+k_{\mathrm{in}, i} \cdot \frac{d f_{\mathrm{AC}}}{d t}, \\
k_{\mathrm{dr}, i}=k_{\mathrm{dr}, i, 0}+k_{\mathrm{dr}} \cdot \frac{\omega_{i}^{2}-\omega_{\min }^{2}}{\omega_{\max }^{2}-\omega_{\min }^{2}},
\end{gathered}
$$

$$
k_{\mathrm{in}, i}=k_{\mathrm{in}, i, 0}+k_{\mathrm{in}} \cdot \frac{\omega_{i}^{2}-\omega_{\min }^{2}}{\omega_{\max }^{2}-\omega_{\min }^{2}},
$$

where $k_{\mathrm{dr}, i}$ and $k_{\mathrm{in}, i}$ represent coefficients of adaptive droop control and virtual inertial control, respectively; then, $k_{\mathrm{dr}, i, 0}$ and $k_{\mathrm{in}, i, 0}$ refer to the initial values of $k_{\mathrm{dr}, i}$ and $k_{\mathrm{in}, i}$, separately; $k_{\mathrm{dr}}$ and $k_{\text {in }}$ denote the slopes of $k_{\mathrm{dr}, i}$ and $k_{i n, i}$, respectively; besides, $f_{A C}$ stands for the frequency of the AC system, which is measured in VSC1 in this article; $f_{\text {ref }}=50 H z, \omega_{\min }=0.70 p u$, and $\omega_{\max }=$ $1.20 p u$.

Additionally, more details about modeling parameters of the whole system, logic of step start-up adaptive inertial and droop control, and the control strategy of VSC stations can be acquired from the literature (Xiong et al., 2021).

\section{PARAMETER DESIGN OF THE STEP START-UP ADAPTIVE INERTIAL AND DROOP CONTROLLER VIA CGO}

\subsection{Fundamental Principle of CGO}

Over the past few decades, omnifarious meta-heuristic algorithms have been proposed, which are triumphantly applied in mathematical and engineering fields thanks to their particular preponderances, e.g., low dependence on models and gradient information of practical problems. Inspired by the fractal configuration and fractal self-similarity issues of the chaos game theory, Talatahari et al. (Talatahari and Azizi, 2020) developed the latest meta-algorithm, namely CGO, whose primary mechanisms can be generalized as follows:

$$
\begin{gathered}
\text { Seed }_{i}^{1}=X_{i}+\alpha_{i, 1} \cdot\left(\beta_{i, 1} \cdot X_{\mathrm{GB}}-\gamma_{i, 1} \cdot X_{\mathrm{MG}, i}\right), \\
\text { Seed }_{i}^{2}=X_{\mathrm{GB}}+\alpha_{i, 2} \cdot\left(\beta_{i, 2} \cdot X_{i}-\gamma_{i, 2} \cdot X_{\mathrm{MG}, i}\right), \\
\text { Seed }_{i}^{3}=X_{\mathrm{MG}, i}+\alpha_{i, 3} \cdot\left(\beta_{i, 3} \cdot X_{i}-\gamma_{i, 3} \cdot X_{\mathrm{GB}}\right), \\
\text { Seed }_{i}^{4}=X_{\mathrm{LB}}+X_{\mathrm{rand}} \cdot\left(X_{\mathrm{UB}}-X_{\mathrm{LB}}\right),
\end{gathered}
$$

where $\operatorname{Seed}_{i}^{1}$, Seed ${ }_{i}^{2}, \operatorname{Seed}_{i}^{3}$, and $\operatorname{Seed}_{i}^{4}$ denote four new solutions for the $i$ th individual $X_{i}$ in the current population; $X_{\mathrm{GB}}$ stands for the current global optimal solution, and $X_{\text {rand }}$ is a random vector which is uniformly distributed in $[0,1] ; X_{\mathrm{MG}, i}$ represents the mean solution of some random individuals in the eligible population; furthermore, $X_{\mathrm{LB}}$ and $X_{\mathrm{UB}}$ are the lower and upper boundaries of problem space, respectively; each of $\beta$ and $\gamma$ is equal to 0 or 1 , while $\alpha$ means a stochastic coefficient for imitating the movement constraints of the solution.

Moreover, more information on CGO can be directly obtained from the literature (Talatahari and Azizi, 2020), e.g., pseudocode, flowchart, corresponding parameter values, etc.

\subsection{Parameter Optimization Framework of the Step Start-Up Adaptive Inertial and Droop Controller Based on CGO}

In this section, a parameter optimization framework of the step start-up adaptive inertial and droop controller combined with the CGO algorithm is provided. This is based upon a suitable 


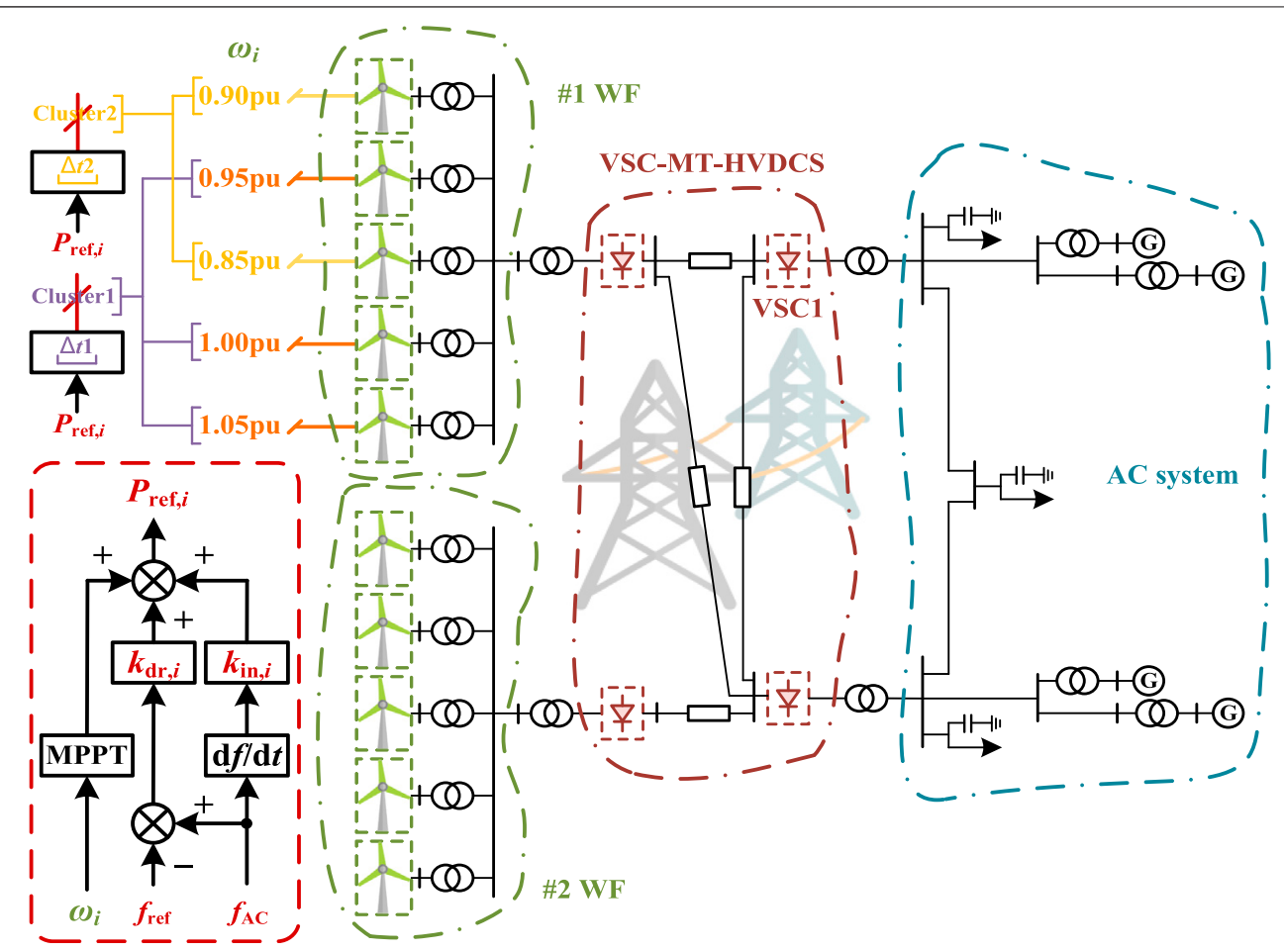

FIGURE 2 | Step start-up adaptive inertial and droop control scheme for a three-area four-terminal VSC-MT-HVDC-based WFs and AC system on the WF level.

TABLE 1 | The range of optimized parameters.

\begin{tabular}{lcccc} 
Parameters & $\mathbf{k}_{\mathrm{dr}, \mathrm{i}, \mathbf{0}}^{\mathbf{M} W / \mathbf{H z}}$ & $\mathbf{k}_{\mathrm{in}, \mathrm{i}, \mathbf{0}} \mathbf{M W} \cdot \mathbf{s} / \mathbf{H z}$ & $\boldsymbol{k}_{\mathrm{dr}} \mathbf{M W} / \mathbf{H z}$ & $\boldsymbol{k}_{\text {in }}(\mathbf{M W} \mathbf{~ s / H z )}$ \\
\hline Upper bound & -120 & -135 & -165 & -155 \\
Lower bound & -20 & -30 & 10 & 20
\end{tabular}

TABLE 2 | Control parameters acquired by various methods under a 200 MW load increase.

Control parameters

Without FSC

Trial-and-error (Xiong et al) (Xiong et al., 2021)

CGO

\begin{tabular}{ccc} 
& \multicolumn{2}{c}{ Cluster $\mathbf{1}$ in $\mathbf{~}$} \\
\hline $\mathbf{k}_{\mathrm{d}, \mathrm{i}, \mathbf{0}}^{1}$ & $\mathbf{k}_{\mathbf{i}, \mathbf{i}, \mathbf{0}}^{\mathbf{1}}$ \\
- & - \\
-60.00 & -60.00 & -1 \\
-21.23 & -134.83 & -1
\end{tabular}

$\begin{array}{cc}\mathbf{k}_{\mathrm{dr}}^{1} & \mathbf{k}_{\mathrm{in}}^{\mathbf{1}} \\ -100.00 & -150.00 \\ -134.78 & -58.85\end{array}$

Cluster 2 in WF 1

\begin{tabular}{cccc}
\hline $\mathbf{k}_{\mathrm{dr}, \mathrm{i}, \mathbf{0}}^{2}$ & $\mathbf{k}_{\mathbf{i}, \mathbf{i}, \mathbf{0}}^{\mathbf{2}}$ & $\mathbf{k}_{\mathrm{dr}}^{2}$ & $\mathbf{k}_{\mathbf{i n}}^{\mathbf{2}}$ \\
- & - & - & $/$ \\
-100.00 & -60.00 & -100.00 & -150.00 \\
-20.00 & -118.63 & -74.64 & -47.18
\end{tabular}

TABLE 3 | FFD, SFD, and TFD obtained by various methods.

Frequency events

A 200-MW load increase

A 300-MW load increase

\section{Approaches}

\section{Without FSC}

Trial-and-error (Xiong et al) (Xiong et al., 2021)

CGO

Without FSC

Trial-and-error (Xiong et al) (Xiong et al., 2021)

CGO
Transient frequency characteristics

\begin{tabular}{lcc}
\hline FFD (Hz) & SFD (Hz) & TFD (Hz) \\
0.2086 & - & - \\
$\mathbf{0 . 1 5 5 8}$ & 0.1364 & 0.1341 \\
0.1592 & $\mathbf{0 . 1 2 9 6}$ & $\mathbf{0 . 1 2 1 7}$ \\
& & - \\
0.2982 & - & 0.1774 \\
$\mathbf{0 . 2 2 3 6}$ & 0.1796 & $\mathbf{0 . 1 6 3 1}$ \\
0.2283 & $\mathbf{0 . 1 7 1 5}$ &
\end{tabular}




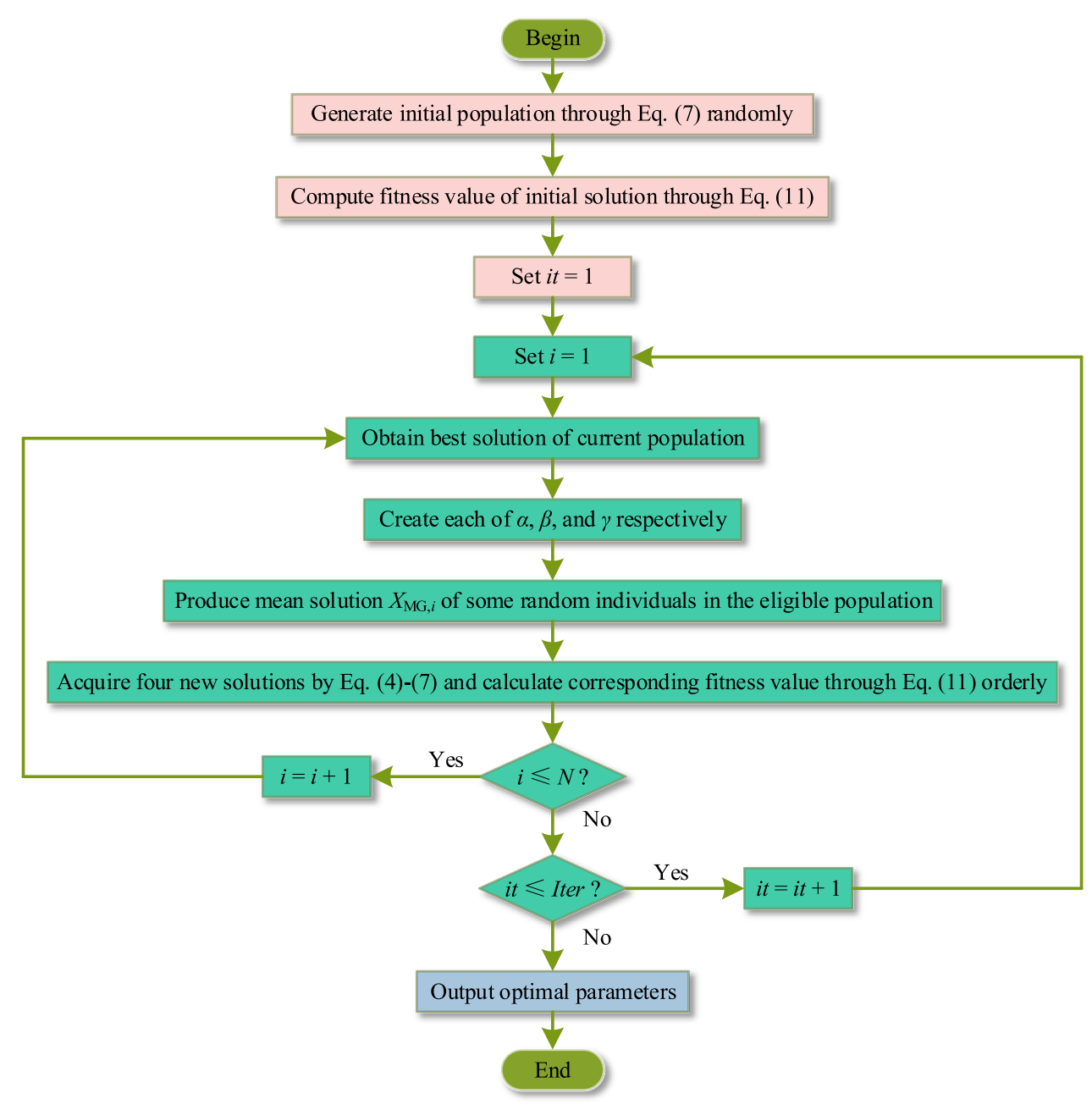

FIGURE 3 | Parameter optimization framework for the step start-up adaptive inertial and droop controller based on CGO.

fitness function which is extremely crucial to obtain a set of optimal control parameters and then efficaciously stabilize the grid frequency under different operation conditions (e.g., load decrease or increase). Hence, three indexes about grid frequency $f_{\mathrm{AC}}$, are considered to construct objective function for the CGO algorithm in this article, i.e., integral of frequency deviation and frequency variation rate $\left(A_{\text {IoFD }}\right.$ and $A_{I O F V R}$, respectively), and maximum frequency deviation point $f_{M F D P}$, which can be successively expressed as follows:

$$
\begin{gathered}
A_{\mathrm{IoFD}}=\int\left|1-f_{\mathrm{AC}}\right| d t, \\
A_{\mathrm{IoFD}}=\int\left|\frac{d f_{\mathrm{AC}}}{d t}\right| d t, \\
f_{\mathrm{MFDP}}=\left\{\begin{array}{ll}
\min \left(f_{\mathrm{AC}}\right), & \text { for load increase } \\
\max \left(f_{\mathrm{AC}}\right), & \text { for load decrease }
\end{array} .\right.
\end{gathered}
$$

And then, each indicator is allocated a weight factor according to the relative importance and order of magnitude. Finally, the objective function is demonstrated in Eq. 11.

$$
\begin{aligned}
F_{\text {obj }}\left(A_{\mathrm{IoFD}}, A_{\mathrm{IoFVR}}, f_{\mathrm{MFDP}}\right)= & \min \left\{\left(10 A_{\mathrm{IoFD}}+100 A_{\mathrm{IoFVR}}\right.\right. \\
& \left.\left.+100 f_{\mathrm{MFDP}}\right) \cdot 50\right\} .
\end{aligned}
$$

Additionally, parameters of the controller for different wind turbines within the same cluster are identically designed for reducing the dimensions of problem space and enhancing the search efficiency of CGO. Therefore, unknown parameters can be further refined as $X=\left[k_{\mathrm{dr}, i, 0}^{1}, k_{\mathrm{in}, i, 0}^{1}, k_{\mathrm{dr}}^{1}, k_{\mathrm{in}}^{1}, k_{\mathrm{dr}, i, 0}^{2}, k_{\mathrm{in}, i, 0}^{2}, k_{\mathrm{dr}}^{2}\right.$, $\left.k_{\mathrm{in}}^{2}\right]$, in which the superscript 1 and 2 stand for cluster 1 and cluster 2, respectively. Besides, the lower and upper limits of optimized parameters are shown in Table 1. Ultimately, the whole parameter optimization procedures of the step start-up adaptive inertial and droop controller with CGO are explicitly exhibited in Figure 3, where $N$ and Iter denote the number of population and maximum iteration, severally.

\section{CASE STUDIES}

In this section, two frequency events, i.e., load increases 200 and $300 \mathrm{MW}$, are employed to evaluate the performance of the proposed 

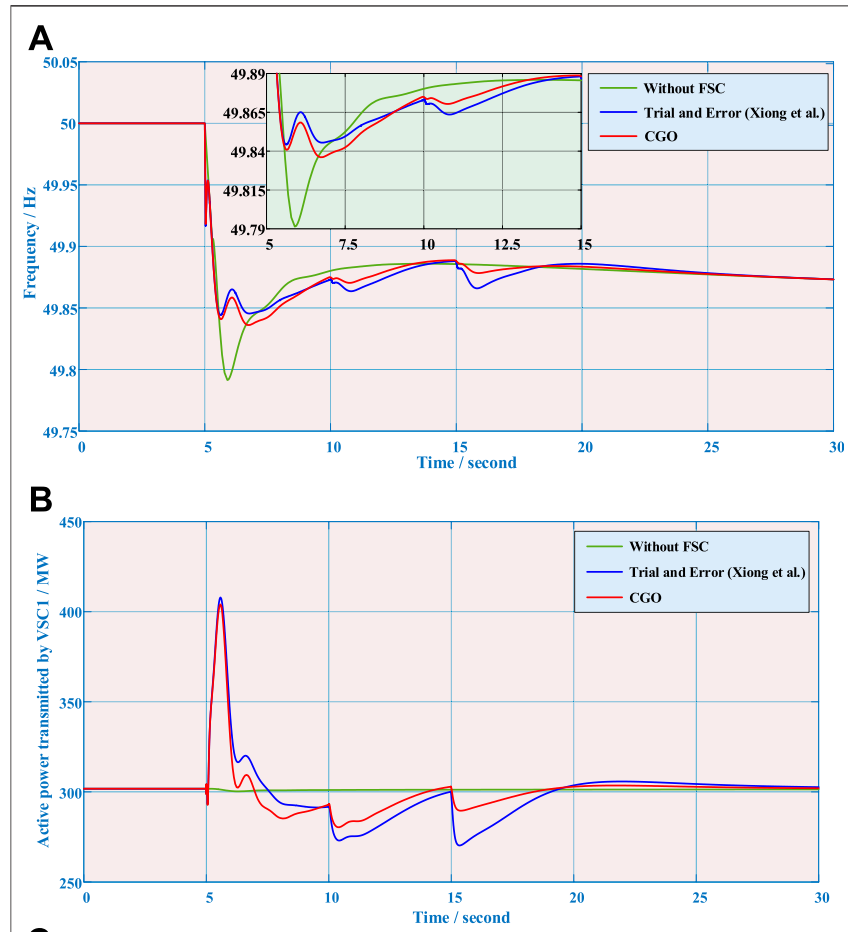

C

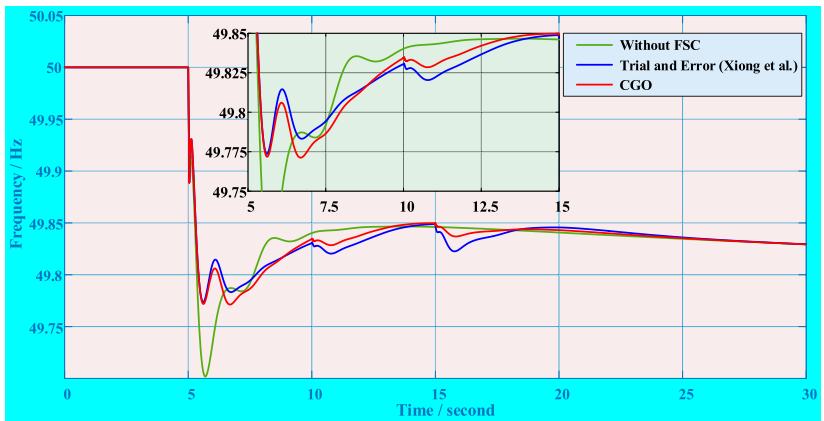

D

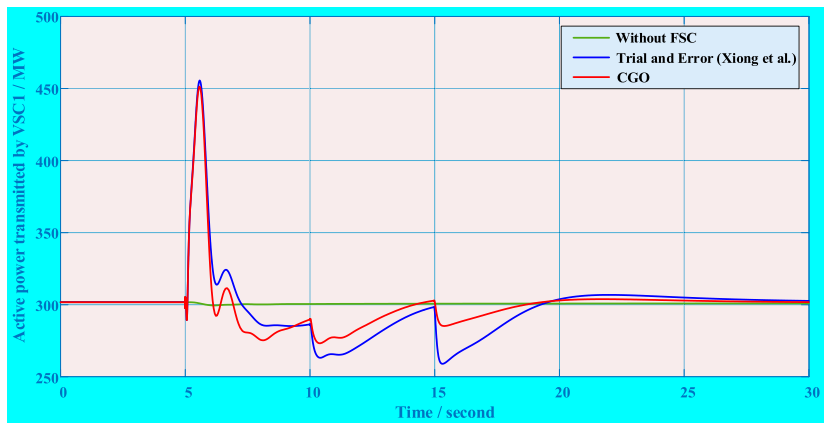

FIGURE 4 | Frequency and transmitted active power of VSC1 under two frequency events: (A) Frequency under a 200-MW load increase; (B) transmitted active power under a 200-MW load increase; (C) Frequency under a 300-MW load increase; (D) transmitted active power under a 300-MW load increase.

method in chapter 3 via a three-area four-terminal VSC-MTHVDC-based WFs and AC system shown in Figure 2. Besides, all case studies are implemented by the MATLAB/Simulink 2019b environment through a personal computer with Intel(R) Core(TM) i5 CPU at $2.9 \mathrm{GHz}$ and $16 \mathrm{~GB}$ of RAM. DAESSC with variable step is selected as the solver. Meanwhile, $N$ and Iter are settled as 5 and 8 , respectively. In particular, all case studies are independently run 10 times to acquire a set of optimal parameters. Furthermore, wind turbine cluster 1 immediately participated to regulate the system frequency after the frequency event appeared $(t=5 \mathrm{~s})$ while wind turbine cluster 2 was put into frequency regulation when rotor speeds of wind turbines in cluster 1 start to recover $(t=10 s)$, i.e., $\Delta t 1=0 s$ and $\Delta t 2=5 s$.

As a result, optimal parameters obtained by three different methods are tabulated in Table 2, i.e., without WFs participating in frequency regulation (without FSC), trial-and-error (Xiong et al.), and the proposed method upon which the symbol " $/$ " indicates no value. Note that the symbol "/" is applicable for the rest tables of this article.

Furthermore, the dynamic process of the measured frequency and active power in VSC1 acquired by different strategies are elaborately portrayed in Figure 4. One can demonstrably see that the frequency fluctuation of CGO is smaller than that of trial-anderror after the frequency event. Particularly, shown in Figures 4A,C, the CGO-based parameter optimization strategy can efficiently restrain the frequency drop (i.e., SFD and TFD) when the rotor speeds of wind turbines in clusters 1 and 2 start to restore at 10 and $15 \mathrm{~s}$, respectively. Meanwhile, compared with without the FSC strategy, Figures 4B,D unquestionably indicate that more active power generated by WFs with FSC is transmitted to the AC system through VSC1 and thus to compensate the power shortage caused by frequency events occurring at $5 \mathrm{~s}$.

Besides, as illustrated in Table 3, three transient frequency characteristics (Delkhosh and Seifi, 2021; Xiong et al., 2021) (i.e., FFD, SFD, and tertiary frequency drop (TFD)) are used here to further concretely quantify and compare the control performance of three designed approaches. Among which the optimal value is highlighted in bold. Both under the 200 and $300 \mathrm{MW}$ load increases, it is pellucidly observed that although FFD with CGO is more than that with trial-and-error, SFD and TFD with CGO are superior to those with trial-and-error, respectively. Specifically, TFDs with CGO only are 0.1217 and $0.1631 \mathrm{~Hz}$ while TFDs with trial-and-error reach up to 0.1341 and $0.1774 \mathrm{~Hz}$ under the 200 and $300 \mathrm{MW}$ load increases, respectively.

\section{CONCLUSION}

In general, the several main conclusions can be described as follows:

- A parameter optimization framework of the step start-up adaptive inertial and droop controller combined with the CGO algorithm is developed to effectively accomplish frequency support via wind farms for the power system;

- A reasonable objective function is carefully designed by considering three essential factors of system frequency, i.e., integral of frequency deviation and frequency variation rate, and maximum frequency deviation point;

- The optimal control parameter is obtained by a three-area fourterminal VSC-MT-HVDC-based WFs and AC system under a $200 \mathrm{MW}$ load increase, which is also applicable under a 
300 MW load increase. Particularly, TFD with CGO merely equals to $0.1631 \mathrm{~Hz}$ while that of trial-and-error reaches up to $0.1774 \mathrm{~Hz}$ under a $300 \mathrm{MW}$ load increase.

The proposed parameter optimization with CGO can significantly alleviate frequency fluctuation and improve power quality under designed frequency events in this article. Thus, it may also be suitable for other frequency events, e.g., WHE, load demand decrease, etc.

\section{DATA AVAILABILITY STATEMENT}

The original contributions presented in the study are included in the article/Supplementary Material, further inquiries can be directed to the corresponding author.

\section{AUTHOR CONTRIBUTIONS}

YC carried out the conceptualization, writing of the original $\mathrm{draft}$, and formal analysis; BY was responsible for supervision,

\section{REFERENCES}

Al Bkoor Alrawashdeh, K., Al-Samrraie, L. a. A., Al-Bsoul, A., Abdullah, A., Lymoon, A., Gul, E., et al. (2021). Investigation of the Influence of Dimensions and Material of the Pipes on the Water Hammer Effect in Microbial Fuel Cells Wastewater Treatment Plants. Sustainable Energ. Tech. Assessments 44, 100990. doi:10.1016/j.seta.2020.100990

Boyle, J., Littler, T., Muyeen, S. M., and Foley, A. M. (2021). An Alternative Frequency-Droop Scheme for Wind Turbines that Provide Primary Frequency Regulation via Rotor Speed Control. Int. J. Electr. Power Energ. Syst. 133, 107219. doi:10.1016/j.ijepes.2021.107219

Chen, J., Yao, W., Zhang, C., Ren, Y., and Jiang, L. (2018). Design of Robust MPPT Controller for Grid-Connected PMSG-Based Wind Turbine via Perturbation Observation Based Nonlinear Adaptive Control. Renew. Energ. 101, 34-51. doi:10.1016/j.renene.2018.11.048

Díaz, H., and Guedes Soares, C. (2020). Review of the Current Status, Technology and Future Trends of Offshore Wind Farms. Ocean Eng. 209, 107381. doi:10.1016/j.oceaneng.2020.107381

Delkhosh, H., and Seifi, H. (2021). Power System Frequency Security index Considering All Aspects of Frequency Profile. IEEE Trans. Power Syst. 36 (2), 1656-1659. doi:10.1109/tpwrs.2020.3047510

Fu, Y., Zhang, X., Hei, Y., and Wang, H. (2017). Active Participation of Variable Speed Wind Turbine in Inertial and Primary Frequency Regulations. Electric Power Syst. Res. 147, 174-184. doi:10.1016/j.epsr.2017.03.001

Gan, W., Ai, X., Fang, J., Yan, M., Yao, W., Zuo, W., et al. (2019). Security Constrained Co-planning of Transmission Expansion and Energy Storage. Appl. Energ. 239, 383-394. doi:10.1016/j.apenergy.2019.01.192

Golpira, H., Seifi, H., Messina, A. R., and Haghifam, M.-R. (2016). Maximum Penetration Level of Micro-grids in Large-Scale Power Systems: Frequency Stability Viewpoint. IEEE Trans. Power Syst. 31 (6), 5163-5171. doi:10.1109/tpwrs.2016.2538083

Gu, M., Meegahapola, L., and Wong, K. L. (2021). Coordinated Voltage and Frequency Control in Hybrid AC/MT-HVDC Power Grids for Stability Improvement. IEEE Trans. Power Syst. 36 (1), 635-647. doi:10.1109/ tpwrs.2020.2983431

Hou, P., Hu, W., Soltani, M., Chen, C., Zhang, B., and Chen, Z. (2017). Offshore Wind Farm Layout Design Considering Optimized Power Dispatch Strategy. IEEE Trans. Sustain. Energ. 8 (2), 638-647. doi:10.1109/tste.2016.2614266

Huang, S., Wu, Q., Liao, W., Wu, G., Li, X., and Wei, J. (2021). Adaptive DroopBased Hierarchical Optimal Voltage Control Scheme for VSC-HVdc and review and editing of the manuscript; $\mathrm{YH}$ conducted formal analysis, software, and supervision; TH was responsible for project administration and resources; $\mathrm{PH}$ conducted investigation and validation; XM carried out the reviewing and editing and methodology; $\mathrm{XH}$ performed visualization and validation.

\section{FUNDING}

This study received funding from the Key Project of China Southern Power Grid Co., Ltd. (YNKJXM20191240). The funder was not involved in the study design, collection, analysis, interpretation of data, the writing of this article, or the decision to submit it for publication.

\section{ACKNOWLEDGMENTS}

The authors gratefully acknowledge the support of Key Project of China Southern Power Grid Co., Ltd. (YNKJXM20191240).

Connected Offshore Wind Farm. IEEE Trans. Ind. Inf. 17 (12), 8165-8176. doi:10.1109/tii.2021.3065375

Jami, M., Shafiee, Q., Gholami, M., and Bevrani, H. (2020). Control of a Supercapacitor Energy Storage System to Mimic Inertia and Transient Response Improvement of a Direct Current Micro-grid. J. Energ. Storage 32, 101788. doi:10.1016/j.est.2020.101788

Kadri, A., Marzougui, H., Aouiti, A., and Bacha, F. (2020). Energy Management and Control Strategy for a DFIG Wind Turbine/fuel Cell Hybrid System with Super Capacitor Storage System. Energy 192, 116518. doi:10.1016/j.energy.2019.116518

Li, R. S., Wong, P., Wang, K., Li, B., and Yuan, F. F. (2020). Power Quality Enhancement and Engineering Application with High Permeability Distributed Photovoltaic Access to Low-Voltage Distribution Networks in Australia. Prot. Control. Mod. Power Syst. 5 (3), 183-189. doi:10.1186/s41601-020-00163-x

Liu, Y., Jiang, H., Peng, J., and Aziz, S. (2020). A Grouping-Based Frequency Support Scheme for Wind Farm under Cyber Uncertainty. IEEE Access 8, 178044-178054. doi:10.1109/access.2020.3016356

Liu, Z., Fan, S., Wang, Y., and Peng, J. (2021). Genetic-algorithm-based Layout Optimization of an Offshore Wind Farm under Real Seabed Terrain Encountering an Engineering Cost Model. Energ. Convers. Management 245, 114610. doi:10.1016/j.enconman.2021.114610

MacDowell, J., Dutta, S., Richwine, M., Achilles, S., and Miller, N. (2015). Serving the Future: Advanced Wind Generation Technology Supports Ancillary Services. IEEE Power Energ. Mag. 13 (6), 22-30. doi:10.1109/mpe.2015.2461331

Meng, Y., Yan, S., Wu, K., Ning, L., Li, X., Wang, X., et al. (2021). Comparative Economic Analysis of Low Frequency AC Transmission System for the Integration of Large Offshore Wind Farms. Renew. Energ. 179, 1955-1968. doi:10.1016/j.renene.2021.07.137

Navalkar, S. T., van Solingen, E., and van Wingerden, J.-W. (2015). Wind Tunnel Testing of Subspace Predictive Repetitive Control for Variable Pitch Wind Turbines. IEEE Trans. Contr. Syst. Technol. 23 (6), 2101-2116. doi:10.1109/tcst.2015.2399452

Pabitra, K. G., and Abhik, B. (2020). Stability Enhancement of Wind Energy Integrated Hybrid System with the Help of Static Synchronous Compensator and Symbiosis Organisms Search Algorithm. Prot. Control. Mod. Power Syst. 5 (2), 138-150. doi:10.1186/s41601-020-00158-8

Renedo, J., Garcia-Cerrada, A., and Rouco, L. (2016). Active Power Control Strategies for Transient Stability Enhancement of AC/DC Grids with VSCHVDC Multi-Terminal Systems. IEEE Trans. Power Syst. 31 (6), 4595-4604. doi:10.1109/tpwrs.2016.2517215

Surinkaew, T., and Ngamroo, I. (2014). Coordinated Robust Control of DFIG Wind Turbine and PSS for Stabilization of Power Oscillations Considering 
System Uncertainties. IEEE Trans. Sustain. Energ. 5 (3), 823-833. doi:10.1109/ tste.2014.2308358

Talatahari, S., and Azizi, M. (2020). Optimization of Constrained Mathematical and Engineering Design Problems Using Chaos Game Optimization. Comput. Ind. Eng. 145, 106560. doi:10.1016/j.cie.2020.106560

Varzaneh, S. G., Gharehpetian, G. B., and Abedi, M. (2014). Output Power Smoothing of Variable Speed Wind Farms Using Rotor-Inertia. Electric Power Syst. Res. 116, 208-217. doi:10.1016/j.epsr.2014.06.006

Vennelaganti, S. G., and Chaudhuri, N. R. (2018). Selective Power Routing in MTDC Grids for Inertial and Primary Frequency Support. IEEE Trans. Power Syst. 33 (6), 7020-7030. doi:10.1109/tpwrs.2018.2854647

Vereide, K., Svingen, B., Nielsen, T. K., and Lia, L. (2017). The Effect of Surge Tank Throttling on Governor Stability, Power Control, and Hydraulic Transients in Hydropower Plants. IEEE Trans. Energ. Convers. 32 (1), 91-98. doi:10.1109/ tec.2016.2614829

Vidyanandan, K. V., and Senroy, N. (2013). Primary Frequency Regulation by Deloaded Wind Turbines Using Variable Droop. IEEE Trans. Power Syst. 28 (2), 837-846. doi:10.1109/tpwrs.2012.2208233

Wang, Q., Li, F., Tang, Y., and Xu, Y. (2019). Integrating Model-Driven and DataDriven Methods for Power System Frequency Stability Assessment and Control. IEEE Trans. Power Syst. 34 (6), 4557-4568. doi:10.1109/ tpwrs.2019.2919522

Wang-Hansen, M., Josefsson, R., and Mehmedovic, H. (2013). Frequency Controlling Wind Power Modeling of Control Strategies. IEEE Trans. Sustain. Energ. 4 (4), 954-959. doi:10.1109/tste.2013.2257898

Wei Yao, W., Lin Jiang, L., Jinyu Wen, J., Qinghua Wu, Q., and Shijie Cheng, S. (2015). Wide-area Damping Controller for Power System Interarea Oscillations: A Networked Predictive Control Approach. IEEE Trans. Contr. Syst. Technol. 23 (1), 27-36. doi:10.1109/tcst.2014.2311852

Wen, J., Liu, J., Long, Y., and Yao, W. (2016). Solution to Short-Term Frequency Response of Wind Farms by Using Energy Storage Systems. IET Renew. Power Generation 10 (5), 669-678. doi:10.1049/iet-rpg.2015.0164

Xiong, L., Liu, X., Zhang, D., and Liu, Y. (2020). Rapid Power Compensation Based Frequency Response Strategy for Low Inertia Power Systems. IEEE J. Emerging Selected Top. Power Electronics 9 (4), 4500-4513. doi:10.1109/ JESTPE.2020.3032063

Xiong, Y., Yao, W., Wen, J., Lin, S., Ai, X., Fang, J., et al. (2021). Two-level Combined Control Scheme of VSC-MTDC Integrated Offshore Wind Farms for Onshore System Frequency Support. IEEE Trans. Power Syst. 36 (1), 781-792. doi:10.1109/tpwrs.2020.2998579

Yang, B., Jiang, L., Yao, W., and Wu, Q. H. (2015). Perturbation Estimation Based Coordinated Adaptive Passive Control for Multimachine Power Systems. Control. Eng. Pract. 44, 172-192. doi:10.1016/j.conengprac.2015.07.012

Yang, D., Jin, Z., Zheng, T., and Jin, E. (2022). An Adaptive Droop Control Strategy with Smooth Rotor Speed Recovery Capability for Type III Wind Turbine
Generators. Int. J. Electr. Power Energ. Syst. 135, 107532. doi:10.1016/ j.ijepes.2021.107532

Yao, Q., Liu, J., and Hu, Y. (2019). Optimized Active Power Dispatching Strategy Considering Fatigue Load of Wind Turbines during De-loading Operation. IEEE Access 7, 17439-17449. doi:10.1109/access.2019.2893957

Zhang, X., Yu, T., Yang, B., Zheng, L., and Huang, L. (2015). Approximate Ideal Multi-Objective Solution $\mathrm{Q}(\lambda)$ Learning for Optimal Carbon-Energy Combined-Flow in Multi-Energy Power Systems. Energ. Convers. Management 106, 543-556. doi:10.1016/j.enconman.2015.09.049

Zhao, X., Lin, Z., Fu, B., and Gong, S. (2021). Research on Frequency Control Method for Micro-grid with a Hybrid Approach of FFR-OPPT and Pitch Angle of Wind Turbine. Int. J. Electr. Power Energ. Syst. 127, 106670. doi:10.1016/ j.ijepes.2020.106670

Zhao, Y., An, A., Xu, Y., Wang, Q., and Wang, M. (2021). Model Predictive Control of Grid-Connected PV Power Generation System Considering Optimal MPPT Control of PV Modules. Prot. Control. Mod. Power Syst. 6 (4), 407-418. doi:10.1186/s41601-021-00210-1

Zhou, B., Fang, J., Ai, X., Yang, C., Yao, W., and Wen, J. (2021). Dynamic Var reserve-constrained Coordinated Scheduling of LCC-HVDC ReceivingEnd System Considering Contingencies and Wind Uncertainties. IEEE Trans. Sustainable Energ. 12 (1), 469-481. doi:10.1109/ TSTE.2020.3006984

Zhu, J., Booth, C. D., Adam, G. P., Roscoe, A. J., and Bright, C. G. (2013). Inertia Emulation Control Strategy for VSC-HVDC Transmission Systems. IEEE Trans. Power Syst. 28 (2), 1277-1287. doi:10.1109/tpwrs.2012.2213101

Conflict of Interest: Author TH, PH, XM, XH are employed by Electric Power Research Institute of Yunnan Power Grid Co., Ltd.

The remaining authors declare that the research was conducted in the absence of any commercial or financial relationships that could be construed as a potential conflict of interest.

Publisher's Note: All claims expressed in this article are solely those of the authors and do not necessarily represent those of their affiliated organizations, or those of the publisher, the editors, and the reviewers. Any product that may be evaluated in this article, or claim that may be made by its manufacturer, is not guaranteed or endorsed by the publisher.

Copyright $\odot 2022$ Chen, Yang, Han, He, He, Meng and He. This is an open-access article distributed under the terms of the Creative Commons Attribution License (CC BY). The use, distribution or reproduction in other forums is permitted, provided the original author(s) and the copyright owner(s) are credited and that the original publication in this journal is cited, in accordance with accepted academic practice. No use, distribution or reproduction is permitted which does not comply with these terms. 


\section{NOMENCLATURE}

$A_{\text {IoFD }}(\mathrm{Hz} \cdot \mathrm{s})$ integral of frequency deviation

$A_{\text {IoFVR }}(\mathrm{Hz})$ integral of frequency variation rate

$f_{A C}(\mathrm{~Hz})$ frequency of AC system

$f_{\text {ref }}(\mathrm{Hz})$ reference value of system frequency

$f_{\text {MFDP }}(\mathrm{Hz})$ maximum frequency deviation point

Iter maximum iteration of CGO

$k_{d r, i}(\mathbf{M W} / \mathbf{H z}), k_{i n, i}(\mathbf{M W} \cdot \mathbf{s} / \mathbf{H z})$ coefficients of adaptive droop control and virtual inertial control, respectively

$k_{d r, i, 0}(\mathbf{M W} / \mathbf{H z}), k_{i n, i, 0}(\mathbf{M W} \cdot \mathbf{s} / \mathbf{H z})$ initial values of $k_{d r, i}$ and $k_{i n, i}$, respectively $k_{d r}(\mathbf{M W} / \mathbf{H z}), k_{i n}(\mathbf{M W} \cdot \mathbf{s} / \mathbf{H z})$ slopes of $k_{d r, i}$ and $k_{i n, i}$, respectively $N$ number of population for CGO

$P_{r e f, i}$ output power reference value of the $i$ th wind turbine in WF1

$P_{m p p, i}$ maximum power point of the $i$ th wind turbine under rotor speed $\omega_{i}$ $\Delta t_{1}, \Delta t_{2}$ (s) time delay of cluster 1 and cluster 2 participating frequency regulation, respectively

$\boldsymbol{\alpha}$ stochastic coefficient for imitating the movement constraints of solution

$\beta, \gamma$ random integer uniformly distributed in ()

$\omega_{i}(\mathrm{rad} / \mathrm{s})$ rotor speed of $i$ th wind turbine

$\omega_{\min }, \omega_{\max }(\mathrm{rad} / \mathrm{s})$ safety range of rotor speed, individually. 